\title{
Peritonitis bacteriana espontánea asociada a bacteriemia por Vibrio cholerae no O1, no $\mathrm{O} 139$
}

\author{
Noelia Ferreira, M. Laura Yantorno, Hugo Mileo, Mónica Sorgentini y Amadeo Esposto.
}

\section{Spontaneous bacterial peritonitis associated with Vibrio cholerae non-O1, non-139 bacteremia}

Vibrio cholerae is a Gram-negative bacilli with curved, comma shape that belongs to the family Vibrionaceae. The antigenic structure consists of a flagellar $\mathrm{H}$ antigen and a somatic $\mathrm{O}$ antigen (used to classify $V$ cholerae in various serogroups). Serogroups 01 and 0139 have caused epidemics of cholera. Vibrio cholerae non-01 non-139 has been isolated from patients with bacteremia, acute secretory diarrhea, dysentery, abdominal pain, nausea, vomiting, fever and cellulitis. Invasive forms such as meningitis, spontaneous bacterial peritonitis (SBP) and encephalitis are uncommon. Immunosuppression and cirrhosis are risk factors for developing invasive disease. This case report describes a cirrhotic patient from Salta, Argentina, consulting for abdominal pain and fever. He was diagnosed with SBP and Vibrio cholerae non-01 non-139 bacteremia. He received antibiotic treatment with third generation cephalosporins for fourteen days with favorable clinical outcome.

Key words: Spontaneous bacterial peritonitis; Vibrio cholerae, bacteraemia.

Palabras clave: Peritonitis bacteriana espontánea, Vibrio cholerae, bacteriemia.

\section{Introducción}

$V$ ibrio cholerae es un bacilo gramnegativo anaerobio facultativo, no esporulado, móvil, con un flagelo polar. Se clasifica por su estructura antigénica de superficie como somático "O" y según la aglutinación con el antisuero específico es subdividido en serogrupos O1/O139 y no O1/O139 o no aglutinables $^{1-3}$.

Vibrio cholerae O1/O139 causa enfermedad diarreica de distinta gravedad. En Argentina reapareció en 1992, causando una epidemia que se inició en la frontera con Bolivia. Se registró el último caso en el año $2005^{4}$.

Los serogrupos no aglutinables causan con mayor frecuencia compromiso extra intestinal. Se han descrito casos de celulitis, fascitis necrosante, endoftalmitis, otitis, meningitis, infecciones de heridas quirúrgicas, peritonitis, bacteriemias, etc. Las formas clínicas invasoras se presentan en pacientes con diferentes grados de inmunocompromiso como diabetes mellitus, cirrosis, enfermedades hematológicas, insuficiencia renal, y pacientes esplenectomizados ${ }^{2,45-10}$. Como fuentes de infección se han identificado al agua y productos marinos contaminados ${ }^{11}$.

En pacientes con cirrosis hepática se observa una alteración de la inmunidad celular, principalmente en la fagocitosis, a lo que se suman factores locales como traslocación y sobrecrecimiento bacteriano. Existe un aumento de la susceptibilidad a infecciones producidas por bacterias, virus y, ocasionalmente, hongos. El score de Child-Pugh alto, las hemorragias gastrointestinales y la hipoalbuminemia han sido descritos como predictores independientes de infección en esta población. La peritonitis bacteriana espontánea (PBE) es la principal complicación infecciosa producida por $V$. cholerae no O1/O139, seguida por infección del tracto urinario y neumonía ${ }^{12}$. Su detección en sangre se asocia a una elevada mortalidad, siendo el segundo sitio más frecuente de aislamiento luego del líquido ascítico. La cirrosis hepática descompensada se ha señalado como un factor de mortalidad independiente para la enfermedad invasora por V. cholerae ${ }^{6}$.

\section{Caso clínico}

Paciente masculino de 64 años de edad, desempleado, oriundo de Salta, Argentina, con residencia en la ciudad de La Plata en los últimos 15 años. No refería viajes a su ciudad de origen. Antecedentes de alcoholismo crónico, várices esofágicas grado II y cirrosis hepática. Fue internado por un cuadro de $24 \mathrm{~h}$ de evolución con mareos, vómitos, disnea clase funcional II y dolor abdominal difuso, moderado, continuo y no irradiado.

$\mathrm{Al}$ ingreso se encontraba en regular estado general, P.A. $95 / 60 \mathrm{mmHg}$, F.C. 80 latidos/min, F.R. 18 ciclos/ min, $\mathrm{T}^{\circ}$ axilar $38^{\circ} \mathrm{C}$, con ictericia, telangectasias generalizadas, hipertrofia parotídea e ingurgitación yugular
Hospital Interzonal General de Agudos San Martín, La Plata. Buenos Aires, Argentina. Servicio de Infectología (NF, MLY, $A E)$.

Servicio de Clínica Médica (HM). Servicio de Microbiología (MS).

Recibido: 15 de febrero de 2012 Aceptado: 24 de junio de 2012

Correspondencia a: Noelia Ferreira Aparicio. noe_ximena@yahoo.com.ar 
sin colapso inspiratorio. Al examen pulmonar presentaba crépitos bibasales, el abdomen era doloroso a la palpación profunda en el flanco y fosa ilíaca izquierdos, con matidez percutoria de concavidad superior, altura hepática por percusión de $5 \mathrm{~cm}$ y edema en las extremidades inferiores. En el examen neurológico se evidenció un temblor "aleteante".

Exámenes de laboratorio: hematocrito 37\%; leucocitos $16.900 / \mathrm{mm}^{3}$ (neutrófilos $80 \%$ ); plaquetas $159.000 / \mathrm{mm}^{3}$; glicemia $1 \mathrm{~g} / \mathrm{l}$; uremia $0,4 \mathrm{~g} / \mathrm{l}$; creatininemia $1,24 \mathrm{mg} / \mathrm{dl}$; bilirrubinemia total $4,13 \mathrm{mg} / \mathrm{dl}$ (bilirrubinemia directa $1,29 \mathrm{mg} / \mathrm{dl}$ ); AST $53 \mathrm{UI} / 1$; ALT $27 \mathrm{UI} / 1$; protrombinemia $42 \%$; albuminemia $2,9 \mathrm{mg} / \mathrm{dl}$; lactato $2,29 \mathrm{mmol} / \mathrm{L}$; score de Child-Pugh C. El estudio de hepatitis viral fue negativo (HBs Ag; HBc AC; Ac VHC no reactivo por método de ELISA).

La ecografía abdominal mostró presencia de ascitis, hígado disminuido de tamaño de contornos lobulados, de ecogenicidad heterogénea y afinamiento de las venas suprahepáticas, hallazgos compatibles con hepatopatía difusa por cirrosis.

Se realizó una paracentesis diagnóstica, cuyo examen citoquímico dio: leucocitos: $28.300 \mathrm{cél} / \mathrm{mm}^{3}$ (neutrófilos $97 \%$, linfocitos 3\%); eritrocitos: 9.800 cél $/ \mathrm{mm}^{3}$, glucosa $1 \mathrm{~g} / \mathrm{l}$, proteínas $19,7 \mathrm{~g} / 1$.

Se indicó cefotaxima $2 \mathrm{~g}$ cada $8 \mathrm{~h}$ iv como tratamiento empírico de PBE. En la tinción de Gram del líquido ascítico y en dos pares de hemocultivos se observaron bacilos gramnegativos. Se evaluó su susceptibilidad a antimicrobianos por difusión, siendo sensible a cefalosporinas de $3^{\circ}$ generación, ciprofloxacina y tetraciclinas.

La cepa fue tipificada como $V$. cholerae mediante pruebas tradicionales. La evaluación del serogrupo y de la presencia de factores de patogenicidad fue realizada en el Instituto de Microbiología Dr. Carlos G. Malbrán de la Ciudad Autónoma de Buenos Aires (Laboratorio de Referencia Nacional) donde se confirmó el serogrupo no O1, no O139.

Se identificaron por RPC los genes que codifican para los siguientes factores de virulencia: TC (toxina colérica): negativa; TCP (factor de colonización o toxin co-regulated pilus): negativo; TTE (toxina termoestable): negativa.

El paciente evolucionó favorablemente, sin fiebre ni dolor abdominal y con disminución del edema periférico. A las $48 \mathrm{~h}$ se intentó realizar paracentesis de control sin lograr obtener líquido ascítico. Completó 14 días de tratamiento con cefotaxima iv y se dio el alta hospitalaria.

No fue posible mediante la anamnesis dirigida establecer el lugar donde el paciente adquirió el $V$. cholerae así como tampoco se detectaron casos sospechosos en el entorno familiar ni en su comunidad cercana. Asimismo vale la pena destacar que residía en un medio urbano, sin contacto con estanques o cursos de agua.

\section{Discusión}

Presentamos el caso de un paciente con cirrosis descompensada, Child- Pugh $\mathrm{C}$, que presentó una $\mathrm{PBE}$ asociada a bacteriemia por $V$. cholerae no $\mathrm{O} 1$ no $\mathrm{O} 139$.

Vibrio cholerae es un bacilo gramnegativo curvo, perteneciente a la familia Vibrionaceae, que se inactiva a ph $<5,5$. Posee los antígenos flagelar $\mathrm{H}$ y somático $\mathrm{O}$, este último permite clasificarlo en serogupos $\mathrm{O} 1 \mathrm{y}$ no $\mathrm{O}^{1,2}$. Existen 206 serogrupos, sólo O1 y O139 se asocian al cuadro clínico de cólera, con el potencial de producir pandemias $^{3}$.

Vibrio cholerae no $\mathrm{O} 1$ no $\mathrm{O} 139$ no aglutina con el antisuero específico del grupo O1. Antiguamente se denominaban "vibriones no aglutinables o no coléricos" pero actualmente se los incluyen dentro de la especie $V$. cholerae.

Como factores de virulencia presenta hemolisinas las cuales participan en el desarrollo de la enfermedad invasora. No elabora toxina colérica ni factor de colonización ${ }^{13,14}$.

Su distribución es mundial y se las aísla preferentemente en estuarios, mares y ríos. No fue posible documentar el foco epidemiológico del caso presentado a pesar del exhaustivo interrogatorio realizado.

En Argentina ocurrieron brotes entre 1992 y 1998 causados por $V$. cholerae $\mathrm{O} 1$ biotipo El Tor pero los cuadros asociados a los serogrupos no O1 no O139 son infrecuentes y con limitada referencia en la literatura científica. La mayoría de los cuadros estuvieron asociados principalmente a cuadros diarreicos y septicemia ${ }^{15}$. Las principales fuentes de transmisión son los alimentos crudos de origen marino (ostras, cangrejos, almejas), la exposición directa de lesiones de la piel al agua de mar y el consumo de agua contaminada ${ }^{3-5}$.

Como factores de riesgo para desarrollar enfermedad invasora se han descrito: cirrosis hepática, inmunodepresión (enfermedades onco-hematológicas, infección por VIH/SIDA, trasplante de precursores hematopoyéticos, etc.), fármacos (antiácidos, corticosteroides, inmunosupresores), diabetes mellitus, insuficiencia cardíaca congestiva, insuficiencia suprarrenal, síndrome nefrótico y hemocromatosis ${ }^{11}$.

En un estudio de Tsong-Yih y cols., los factores pronósticos independientes de mortalidad para enfermedad invasora por $V$. cholerae no O1/139 fueron cirrosis hepática grave $(\mathrm{p}=0,003)$, enfermedades malignas $(\mathrm{p}=$ $0,034)$ y uso de corticosteroides $(p=0,011)^{16}$.

Clínicamente puede presentarse en forma asintomática o bien con manifestaciones gastrointestinales tales como diarrea acuosa o disentería, dolor abdominal, náuseas y vómitos. La fiebre, signo infrecuente en el cólera, puede detectarse en 43 a $71 \%$ de los casos. También puede causar infecciones de piel y tejidos blandos como celulitis $(20 \%)$ y en algunas ocasiones flictenas hemorrágicas en extremidades inferiores ${ }^{6-10,17}$ 
Las formas clínicas graves y diseminadas se observan con mayor frecuencia en pacientes inmunocomprometidos y se manifiestan como bacteriemia, peritonitis, colangitis y fascitis necrosante.

La bacteriemia es el segundo sitio de aislamiento donde se observa una alta mortalidad, hasta $60 \%$ en pacientes cirróticos $^{9,12,14,18-20}$. En el caso presentado, a diferencia de lo descrito en la literatura científica, evolucionó rápida y favorablemente con el tratamiento antimicrobiano parenteral. En algunas series ${ }^{20}$, la bacteriemia es la principal presentación clínica, alcanzando casi $60 \%$ en este tipo de pacientes y la mitad de estos episodios cursa con PBE en forma secundaria.

Las infecciones en pacientes con cirrosis hepática presentan una alta morbi-mortalidad. Estos individuos presentan un inmunocompromiso de origen multifactorial (déficit en la actividad del sistema retículo endotelial, sistema del complemento, opsonización y fagocitosis) sumado a factores locales como traslocación y sobrecrecimiento bacteriano ${ }^{12-21}$.

El diagnóstico se realiza a través del aislamiento de $V$. cholerae en hemocultivos, coprocultivos, cultivo o biopsia de piel y tejidos blandos o cultivo de líquido ascítico, según su forma de presentación.

No hay evidencia clara de cuál es el tratamiento antimicrobiano más efectivo, si bien se recomienda el uso de cefalosporinas de tercera generación como monoterapia o en combinación con tetraciclinas o fluoroquinolonas ${ }^{8,22,23}$.
En nuestro paciente el tratamiento con cefalosporinas de tercera generación obtuvo una rápida y favorable respuesta.

\section{Resumen}

Vibrio cholerae es un bacilo gramnegativo, curvo y móvil, perteneciente a la familia Vibrionaceae, que presenta antígenos flagelares $\mathrm{H}$ y somático $\mathrm{O}$; este último permite clasificarlo en numerosos serogrupos. Los serogrupos O1 y O139 han causado epidemias de cólera.

Vibrio cholerae serogrupo no O1, no O139 es no aglutinable con el antisuero específico y se manifiesta clínicamente como bacteriemias, diarrea acuosa, disentería, dolor abdominal, náuseas, vómitos, fiebre y celulitis. Raramente se describen formas invasoras tales como meningitis, peritonitis bacteriana espontánea (PBE) y cerebritis, entre otras. Dentro de los factores de riesgo para desarrollar enfermedad invasora se encuentran la inmunodepresión y la cirrosis hepática.

Comunicamos el caso de un paciente procedente de la provincia de Salta, Argentina, con antecedentes de cirrosis hepática, que consulta por dolor abdominal y fiebre, en el que se diagnóstica PBE asociada a bacteriemia por Vibrio cholerae no 01, no 0139. Recibió tratamiento con cefalosporinas de tercera generación iv, por catorce días con una evolución clínica favorable.

\section{Referencias bibliográficas}

1.- Seas C, Gotuzzo E. Chapter 214: Vibrio cholerae. Mandell, Douglas and Bennett's. Principles and Practice of Infectious Diseases. $7^{\text {th }}$ ed. 2010. Churchill Livingstone Elsevier, Philadelphia, PA, USA, pp. 2777-86.

2.- Kontoyiannis D P, Calia K E, Basgoz N, Calderwood S B. Primary septicemia caused by Vibrio cholerae non-O1 acquired on Cape Cod, Massachusetts. Clin Infect Dis 1995; 21: 1330-3.

3.- Ghassemi S, García-Tsao G. Prevention and treatment of infections in patients with cirrhosis. Best Pract Res Clin Gastroenterol 2007; 21: 77-93.

4.- Boletín epidemiológico periódico del Ministerio de Salud. Presidencia de la Nación. Revisión de 1998-2010. Disponible en: http://www.msal.gov. $\mathrm{ar} / \mathrm{htm} /$ site/boletin-vigilancia.asp (Consultado: 30 de noviembre de 2010).

5.- $\quad$ Namdari H, Klaips C R, Hughes J L. A cytotoxin-producing strain of Vibrio cholerae non-O1, non-O139 as a cause of cholera and bacteremia after consumption of raw clams. J Clin Microbiol 2000; 38: 3518-9.
6.- Eltahawy A T, Jiman-Fatani A, Al-Alawi M M. A fatal non-O1 Vibrio cholerae septicemia in a patient with liver cirrhosis. Saudi Med J 2004; 25: $1730-1$.

7.- González Fraga S, Pichel M, Costagliola M, Cecilia M, Jurquiza D, Peressutti S, et al. Environment and virulence factors of Vibrio cholerae strains isolated in Argentina. J Appl Microbiol 2007; 103: 2448-56.

8.- Fernández J M, Serrano M, De Arriba J J, Sánchez M V Escribano , Ferreras Bacteremic cellulitis caused by Non-O1, Non-O139 Vibrio cholerae: report of a case in a patient with hemochromatosis. Diagn Microbiol Infect Dis 2000; 37: 77-80.

9.- Morris J G Jr. Non-O group 1 Vibrio cholerae: a look at the epidemiology of an occasional pathogen. Epidemiol Rev 1990; 12: 179-91.

10.- Choi S M, Lee D G, Kim M S, Park Y H Kim Y , Lee S, et al. Bacteremic cellulitis caused by non-O1, non- O139 Vibrio cholerae in a patient following hematopoietic stem cell transplantation. Bone Marrow Transplant 2003; 31: 1181-2.

11.- Lin C J, Chiu C T, Lin D Y, Sheen I S,
Lien J M Non-O1 Vibrio cholerae bacteremia in patients with cirrhosis: 5 -yr experience from a single medical center. Am J Gastroenterol 1996; 91: 336-40.

12.- Tandon P, García-Tsao G. Bacterial infections, sepsis, and multiorgan failure in cirrhosis. Semin Liver Dis 2008; 28: 26-42.

13.- Anderson A M, Varkey J B, Petti C A, Liddle R A Frothingham R, Woods CW Non-O1 Vibrio cholerae septicemia: case report, discussion of literature, and relevance to bioterrorism. Diagn Microbiol Infect Dis 2004; 49: 295-7.

14.- Crump J A, Bopp C A, Greene K D, Kubota KA, Middendorf R L, Wells J G, et al. Toxigenic Vibrio cholerae serogroup O141-associated cholera-like diarrhea and bloodstream infection in the United States. J Infect Dis 2003; 187: 866-8.

15.- González Fraga S, Villagra de Trejo A, Pichel M, Figueroa S, Merletti G, Caffer MI, et al. Caracterización de aislamientos de Vibrio cholerae no-O1, no-O139 asociados a cuadros de diarrea. Rev Argent Microbiol 2009; 41: 11-9.

16.- Ou T Y Liu J W, Leu H S. Independent prognostic factors for fatality in patients 
with invasive Vibrio cholerae non-O1 infections. J Microbiol Immunol Infect 2003; 36: 117-22.

17.- Briceño L, Puebla C, Guerra F, Jensen D, Núñez H, Ulloa MT, et al. Septicemia fatal causada por Vibrio cholerae no-O1, no-O139 hemolítico en Chile. Rev Med Chile 2009; 137 . 1193-6.

18.- Safrin S, Morris J G Jr, Adams M, Pons Jacobs R, Conte J E J N Non-O1 Vibrio cholerae bacteremia: case report and review. Rev Infect Dis $1988 ; 10: 1012-7$.
19.- Ichinose Y, Ehara M, Shimizu T, Ohmagari T, Kaku M Hara K, et al. The characterisation of Vibrio cholerae Non-O1 strain causing perforative pan-peritonitis. Kansenshogaku Zasshi 2001; 75: 696-702.

20.- Ko W C, Chuang Y C, Huang G C, Hsu S Y. Infections due to non-O1 Vibrio cholerae in southern Taiwan: predominance in cirrhotic patients. Clin Infect Dis 1998; 27: 774-80.

21.- Ghassemi S, García-Tsao G. Prevention and treatment of infections in patients with cirrhosis. Best Pract Res Clin Gastroenterol 2007; 21: 77-93.

22.- Patel N M, Wong M, Little E, Ramos AX, Kolli G, Fox K M, et al. Vibrio cholerae non-O1 infection in cirrhotics: case report and literature review. Transpl Infect Dis 2009; 11: 54-6.

23.- El-Hiday A H, Khan F Y, Maslamani M, El Shafie S. Bacteremia and spontaneous bacterial peritonitis due to Vibrio cholerae (non-O1 non-O139) in liver cirrhosis. Indian J Gastroenterol 2006; 25: 107. 